\title{
Combined chemotherapy and radiation therapy for central nervous system germ cell tumors: preliminary results of a Phase II study of the Japanese Pediatric Brain Tumor Study Group
}

Masao Matsutani, M.D., Yukitaka Ushio, M.D., Hiroshi Abe, M.D., Junko Yamashita, M.D., Soichiro Shibui, M.D., Takamitsu Fujimaki, M.D., Kintomo Takakura, M.D., Kazuhiro Nomura, M.D., Ryuichi Tanaka, M.D., Masashi Fukui, M.D., Takashi Yoshimoto, M.D., Toru Hayakawa, M.D., Tadashi Nagashima, M.D., Kaoru Kurisu, M.D., Takamasa Kayama, M.D., and the Japanese Pediatric Brain Tumor Study Group

Departments of Neurosurgery, Saitama Medical School, Moroyama; Kumamoto University Hospital, Kumamoto; Hokkaido University Hospital, Sapporo; Kanazawa University Hospital, Kanazawa; National Cancer Center Hospital, Tokyo; University of Tokyo Hospital, Tokyo; Tokyo Women's University of Medicine, Tokyo; Niigata University Hospital, Sendai; Osaka University Hospital, Osaka; Teikyo School of Medicine Ichihara Hospital, Ichihara; Hiroshima University Hospital, Hiroshima; and Yamagata University Hospital, Yamagata

The authors conducted a multiinstitutional phase II study to establish a postsurgical combined chemotherapy and radiation therapy regimen for patients with primary germ cell tumors of the brain.

After surgical debulking of the tumor and histological verification, patients were divided into three therapeutic groups: good prognosis, intermediate prognosis, and poor prognosis. Patients received two kinds of chemotherapy (three courses) prior to receiving radiation therapy: carboplatin-etoposide combination ([CARB-VP]: carboplatin $450 \mathrm{mg} / \mathrm{m}^{2}$ on Day 1, etoposide $150 \mathrm{mg} / \mathrm{m}^{2}$ on Days $1-3$ ) or ifosphamide-cisplatin-etoposide combination ([ICE]: ifosphamide $900 \mathrm{mg} / \mathrm{m}^{2}$, cisplatin $20 \mathrm{mg} / \mathrm{m}^{2}$, and etoposide $60 \mathrm{mg} / \mathrm{m}^{2}$ on Days 1-5). Patients in the good prognosis group (those with germinomas) were treated with CARB-VP followed by local radiation therapy (24 Gy). Patients in the intermediate prognosis group received CARB-VP followed by local radiation therapy (50 Gy); they received five additional chemotherapy treatments. Patients in the poor prognosis group received ICE followed by whole craniospinal radiation therapy; they also received five additional chemotherapy treatments. Eighty-two patients were evaluated. For the 56 patients with germinomas, a $93 \%$ rate of complete remission after treatment was achieved. The remission rate was $76 \%$ for 21 patients in the intermediate prognosis group, and no recurrence was detected during a median follow-up period of 2.6 years. In the group of five patients with poor prognosis, the disease in three patients progressed during chemotherapy or radiation therapy and they died within 6 months. There were no serious complications in the surviving patients. 
The authors found their treatment protocols to be currently effective for patients with germinomas and those with an intermediate prognosis.

\section{Key Words * brain tumor * germ cell tumor * radiation therapy * chemotherapy}

Germ cell tumors (GCTs) are characterized by subtypes with different histology that show different responses to treatment. Excellent treatment results have been obtained for mature teratomas by curative surgery and for pure germinomas by effective radiation therapy. However, the remaining tumor types (nongerminomatous tumors) have proved refractory to conventional treatments with surgery and irradiation. After the establishment of effective combination chemotherapy with cisplatin for gonadal GCTs, $[2,5]$ nongerminomatous GCTs of the brain became candidates for chemotherapy. Investigators in some trials in which chemotherapy is used have suggested its effectiveness for these tumors, $[9,11,17]$ but the effect has not yet been evaluated in a large study group.

The University of Tokyo Study Group analyzed the treatment results of 134 histologically verified cases, which clearly showed that outcome differs among histological subtypes.[14] They divided patients with intracranial GCTs into three therapeutic groups: good prognosis, intermediate prognosis, and poor prognosis (Table 1) and proposed a treatment guideline appropriate for each group.

Based on their proposal, we conducted a multiinstitution phase II study. The aim of the study was to establish a postsurgical combination therapy in which chemotherapy and radiation therapy were used to make possible a better quality of life for patients with germinomas and a longer survival time for patients with nongerminomatous tumors. We now report our preliminary results of the phase II study in 82 newly diagnosed patients with histologically verified central nervous system GCTs.

\section{CLINICAL MATERIAL AND METHODS}

\section{Study Design}

The study group was organized by 14 neurosurgical clinics in Japan. The treatment strategy of the study was to surgically debulk the tumor and verify the histological composition. This was followed by preirradiation chemo- and radiation therapy. The actual goals of the study were determined for each group.

Germinoma. Although radiation therapy has produced an excellent 10 -year survival rate of more than $80 \%$ of patients with germinomas, approximately $10 \%$ experienced tumor recurrence or dissemination. $[4,14,24]$ In addition, a radiation dose of $50 \mathrm{~Gy}$ delivered to the whole brain in children produced mental retardation and dysfunction of the pituitary gland in later life.[7,12] Therefore, by combining chemotherapy with radiation therapy, we intended to produce a synergistic effect for the tumor and to reduce the radiation dose and target volume in patients with germinoma.

Intermediate Prognosis Group. We adopted a combination chemotherapy regimen with carboplatin-etoposide to prolong the survival time of patients in this group. The regimen was proved effective for tumors in this group in a clinical trial conducted by the University of Tokyo Study Group. $[13,14]$

Poor Prognosis Group. Because previous methods for delivering chemotherapy (carboplatin-etoposide combination or cisplatin-vinblastine-bleomycin combination) have failed to prolong patient survival 
time,[14] we adopted an ifosphamide combination that was shown to be effective in a clinical trial by Hokkaido University Study Group (unpublished data).

After surgical debulking of the tumor and verification of histological composition, patients were divided into three therapeutic groups: good prognosis, intermediate prognosis, and poor prognosis (Table 1). Postsurgically, patients received chemotherapy first, followed by radiation therapy.

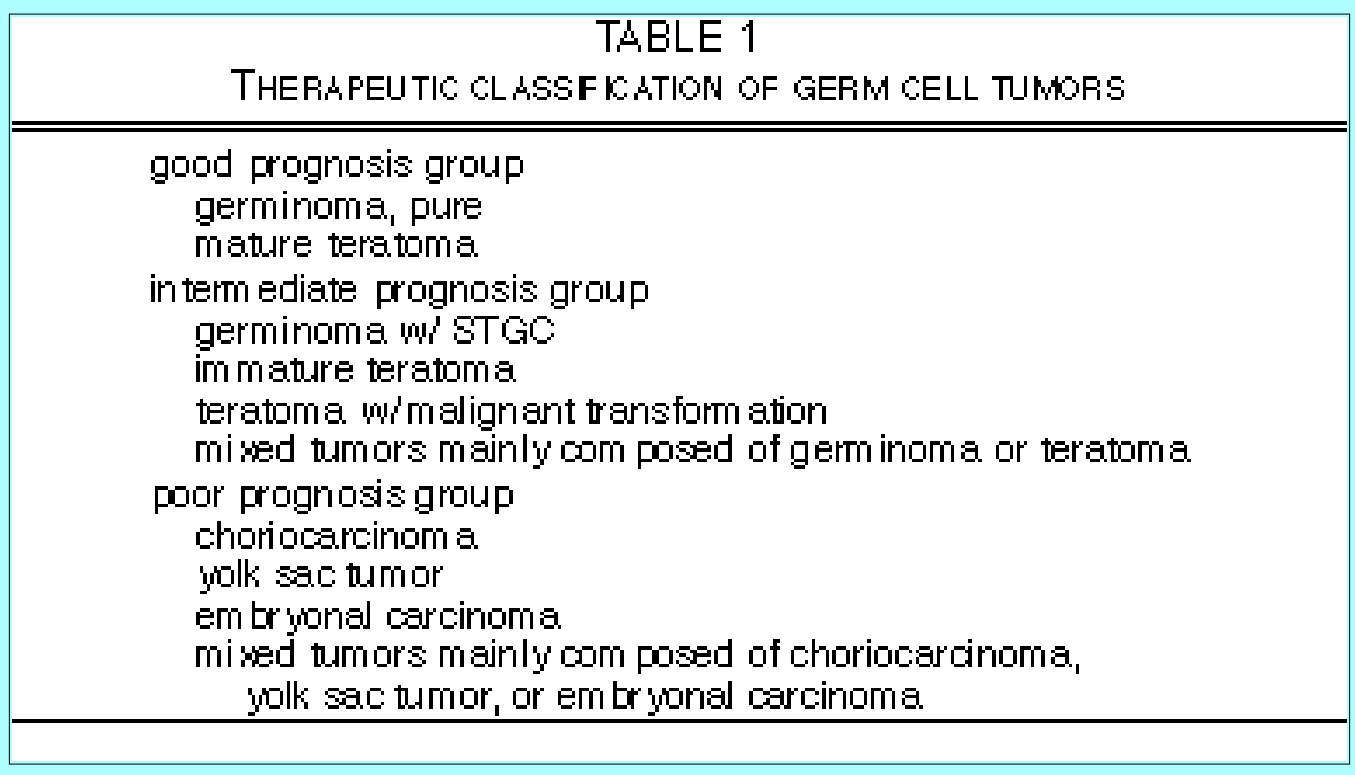

\section{Delivery of Chemotherapy}

Each chemotherapy regimen was applied to selected histological subtypes: a carboplatin-etoposide combination ([CARB-VP] consisting of carboplatin $450 \mathrm{mg} / \mathrm{m}^{2}$ of body surface on Day 1; and etoposide, $150 \mathrm{mg} / \mathrm{m}^{2}$ for 3 consecutive days from Days 1 to 3; this regimen was repeated every 4 weeks for three courses as an induction therapy) and an ifosphamide-cisplatin-etoposide combination ([ICE] consisting of ifosphamide $900 \mathrm{mg} / \mathrm{m}^{2}$, cisplatin $20 \mathrm{mg} / \mathrm{m}^{2}$, and etoposide $60 \mathrm{mg} / \mathrm{m}^{2}$ for 5 consecutive days from Days 1 to 5; this regimen was repeated every 4 weeks for three courses as an induction therapy).

\section{Radiation Therapy}

Radiation therapy in which a 4 MV linear accelerator x-ray was delivered to the tumor area, to a generous local area, or to the whole brain and the whole spine. Radiation doses delivered to a generous local field encompassed the tumor site and the third and lateral ventricles, as well as the sellar and pineal regions.

\section{Treatment Protocols}

Patients with germinoma received CARB-VP followed by local radiation doses ( $24 \mathrm{~Gy}$ ) delivered to the tumor site. A dose of $24 \mathrm{~Gy}$ was chosen from the data regarding the maximum dose to protect the anterior pituitary gland in children from radiation damage.[20] Patients in the intermediate prognosis group received CARB-VP followed by a radiation dose of $30 \mathrm{~Gy}$ delivered to a generous local field and $20 \mathrm{~Gy}$ to the tumor site. They received additional CARB-VP chemotherapy for a total of five times every 3 to 4 months. Patients in the poor prognosis group received ICE followed by whole-brain and spinal radiation therapy with a dose of $30 \mathrm{~Gy}$ and a 30-Gy boost delivered to the generous local field. They received additional ICE a total of five times every 3 to 4 months. 


\section{Evaluation of the Treatment}

To determine the response to therapy, we measured the area of the enhanced mass lesion in each slice of contrast-enhanced magnetic resonance (MR) images before and after the treatment. The responses were divided into four groups. A complete response was recorded when there was no visible tumor and no secondary changes, such as deformity or shift of the ventricles as a result of the tumor removal. More than $50 \%$ reduction of the tumor area on MR imaging without an increase in secondary changes was recorded as a partial response. If there was a less than 50\% reduction of the tumor area on MR imaging or a less than $25 \%$ increase in the tumor area without an increase in secondary changes, the patient was considered to be in stable condition. The tumor was recorded as having progressed if there was a more than $25 \%$ increase in the tumor area accompanied by an increase in secondary changes caused by the tumor. In cases with complete and partial responses the treatment was recorded as effective, and the response rate was calculated as the percentage of cases with complete and partial responses among all cases with tumors visible on MR images. When the tumor was not visible on MR images because of extensive surgical removal, the volume reduction was not evaluated; these cases were excluded from the calculation of the response rate.

To evaluate the effectveness of our study protocol, we compared our treatment results to those of reported cases treated by radiation therapy alone. The survival rate was calculated from the date of surgery to the date of death by using the Kaplan-Meier method.

\section{Patient Population}

One hundred fourteen patients were registered in this trial since April 1995; nine of these patients with germinomas who had obtained excellent results with chemotherapy refused radiation therapy. In the end, 105 patients were enrolled in this trial: 86 males $(81.9 \%)$ and 19 females $(18.1 \%)$ with a mean age of 17 years (range 2-39 years). Only three patients (2.9\%) were younger than 6 years of age, and four patients (3.8\%) were older than 33 years of age; $78.4 \%$ of the patients were between 6 and 24 years of age. Forty tumors $(38.1 \%)$ were located in the pineal region, $27(25.7 \%)$ in the neurohypophysis, seven $(6.7 \%)$ in the basal ganglia, one in the hypothalamus, one in the thalamus, one in the cerebellum; $28(26.7 \%)$ tumors developed at multiple sites.

Of the 105 patients, 82 completed the initial treatment, which consisted of surgery, chemotherapy, and radiation therapy, and they were then evaluated according to their initial response to the treatment. Histological examination determined the diagnoses of germinoma in 56 patients (68.3\%), germinoma with syncytiotrophoblastic giant cells (STGCs) in eight (9.8\%), immature teratoma in three (3.7\%), teratoma with malignant transformation in two (2.4\%), embryonal carcinoma in one $(1.2 \%)$, and mixed tumor in $12(14.6 \%)$. Patients were reclassified into three groups according to their prognosis: 56 were included in the good prognosis group (germinoma), 21 in the intermediate prognosis group, and five in the poor prognosis group. In this discussion, we apply the term "malignant teratoma" to immature teratomas that contained immature embryonic structures of malignant character and to teratomas with malignant transformation.

\section{RESULTS}

Tables 2 and 3 summarize the response to chemotherapy and radiation therapy. 


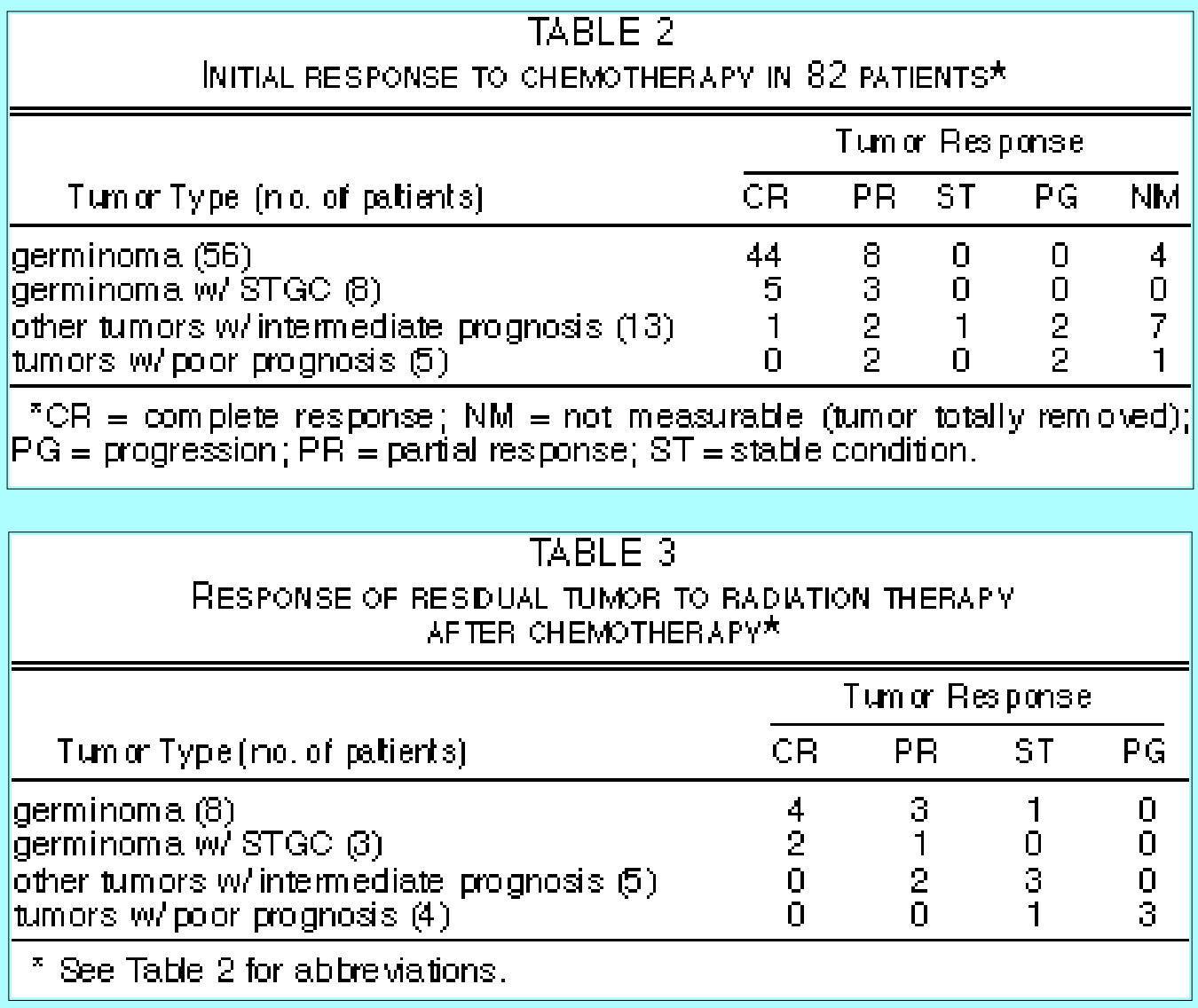

\section{Good Prognosis Group}

Of the 56 patients with germinoma, macroscopic total removal was achieved in four patients. After chemotherapy, tumors completely disappeared in 44 patients; the rate of complete response was $84.6 \%$ (44 of 52 patients). Of the remaining eight patients whose tumors partially responded to chemotherapy, four achieved complete response after radiation therapy, three showed further tumor shrinkage, and one was unchanged. After radiation therapy, these four residual tumors were small, with a diameter of less than $2 \mathrm{~mm}$ on MR imaging. An overall tumor-free rate after the initial treatment with surgery, chemotherapy, and radiation therapy was $92.9 \%$ (52 of 56 patients). With a median follow-up period of 2.1 years, we encountered only one recurrence outside the irradiated area.

We also evaluated the effect of chemotherapy in nine patients with germinoma who dropped out of the study protocol because they refused radiation therapy after chemotherapy. Eight patients (89\%) showed complete tumor disappearance; three tumors recurred within 1 year. These patients were successfully retreated with radiation therapy.

\section{Intermediate Prognosis Group}

In eight patients with germinoma with STGCs, total removal of the tumor was not attempted; all patients were evaluated after receiving chemotherapy. Complete disappearance of the tumor was observed in five patients $(62.5 \%)$, and a partial response was achieved in three. Two of these three patients later achieved complete response and the third achieved partial response. The total tumor-free rate after the initial treatment was $87.5 \%$.

There were 13 other patients included in this group: six had mixed tumors with germinoma and teratoma, two had mixed tumors consisting mainly of germinoma or teratoma, and five had malignant teratomas. 
Total surgical removal of the tumor was performed in seven patients; chemotherapy resulted in a response rate of $50 \%$ (one complete and two partial responses, one stable condition, and two progressions) in the six patients with visible tumors after surgery. After radiation therapy, five of the visible tumors responded: two tumors partially responded and three remained in stable condition. The total tumor-free rate after the initial treatment was $61.5 \%$.

In the whole group of 21 patients with intermediate prognosis, the total tumor-free rate was $76 \%$ and no recurrences were encountered within a median follow-up period of 2.6 years.

\section{Poor Prognosis Group}

This group consisted of one patient with embryonal carcinoma and four patients with mixed tumors, of which the main component was embryonal carcinoma. Total surgical removal of the tumor was performed in one patient with mixed tumor; in the remaining four patients, two partial responses were observed and two tumors progressed after chemotherapy. Three patients experienced tumor progression during chemotherapy and died of disease within 6 months.

\section{Side Effects}

Almost all the patients manifested myelosuppression, especially severe leukocytopenia of less than $2000 / \mathrm{mm}^{3} 5$ to 10 days after the chemotherapy; patients recovered from myelosuppression uneventfully after administration of granulocyte colony-stimulating factor.

\section{DISCUSSION}

In the treatment of GCTs in the brain, radiation therapy with a median tumor dose of 50 Gy was used postoperatively in all patients except for those in whom a total removal of mature teratomas was achieved. Jennings, et al.,[8] have analyzed the survival time of 216 previously reported patients who received conventional treatment with surgery and radiation therapy, noting that most patients with nongerminomatous tumors failed to survive longer than 3 years. The Japanese Intracranial Germ Cell Tumor Study Group[10] has also reported a median survival of 18 months and a rate of dissemination through the cerebrospinal fluid or hematogeneous metastasis of $45 \%$ in 33 patients with nongerminomatous tumors who received postoperative radiation therapy.

Since the beginning of the 1980s, following the development of chemotherapy for testicular GCTs,[2,5] chemotherapy has been delivered as an adjuvant therapy in patients with nongerminomatous GCTs in the brain. The authors of the first trial in which a cisplatin-vinblastine-bleomycin combination was used for intracranial nongerminomatous tumors in Japan reported a 2-year survival rate of $67.7 \%$ in 30 patients, which was slightly better than the $46.5 \%$ in patients who received radiation therapy alone.[10] Thereafter, a cisplatin-etoposide combination and carboplatin-etoposide combination were used in the phase II study by the Japanese Intracranial Germ Cell Tumor Study Group.[31] These trials obtained slightly better results in nongerminomatous tumors but failed to obtain any significant benefit.

The treatment analysis of the Tokyo University Study Group clarified that treatment results differ among histological subtypes, especially among mixed tumors.[14] Their precise analysis of the histological elements comprising mixed tumors revealed that the chemotherapy regimens of cisplatin or carboplatin combinations administered in their study were effective for tumors with intermediate prognosis but not for tumors with poor prognosis.[13] A high incidence of local failure (73.5\%) led to treatment failure in these tumors, suggesting the need for more aggressive, intensive treatments with chemotherapy and 
radiation therapy other than carboplatin-etoposide combination directed toward the primary site.

This multiinstitutional phase II study was based on our clinical experiences in Japan and was designed first to give patients with germinoma a better quality of life, by decreasing the dose and the volume of irradiation, and higher cure rate (more than $90 \%$ ) by using a combination chemotherapy. The second objective of the study was to achieve a 5-year survival rate of more than $50 \%$ for patients with an intermediate prognosis, and the third objective was to achieve a 3-year survival rate of more than 50\% for patients in the poor prognosis group.

In patients with germinomas, the rate of complete response was $84.6 \%$ (44 of 52 patients) after administration of chemotherapy. The rate was nearly the same in eight of nine patients who had received chemotherapy alone, whose complete response rate was $89 \%$. Three tumors in these nine patients recurred within 1 year. These findings indicate that chemotherapy with carboplatin and etoposide alone cannot control germinoma without radiation therapy. The treatment results, providing a total tumor-free rate of $92.9 \%$ and only one recurrence during a median follow-up period of 2.1 years, are currently satisfactory, because all the residual visible tumors in four patients were very small (diameter $<2 \mathrm{~mm}$ ), and the recurrence rate reported is usually 5 to $10 \%$. We can now say that whole-brain irradiation is no longer necessary for germinoma and that a reduced dose of $24 \mathrm{~Gy}$ delivered to the tumor site is sufficient to control germinoma in combination with chemotherapy. Dattoli and Newall,[4] Shibamoto, et al.,[25] and Sawamura, et al.,[23] have also recommended local irradiation for germinoma. Matsutani, et al.,[14] have insisted that local irradiation contributes to a better quality of life (education and employment) in $83 \%$ of long-surviving patients with germinoma, whereas Jenkin, et al.,[7] have reported education difficulty in children with germinoma treated by whole-brain radiation therapy. As for the possibility of protecting anterior pituitary function and a long-term control rate for the disease with our treatment, longer follow-up study is necessary.

In germinomas with STGC, a complete response was observed in five (62.5\%) of eight patients; the complete response rate was lower than that of germinoma. The reported higher recurrence rate of 40 to $50 \%$ with a median time of 3 years for this tumor[14,28,30] might correlate with this lower response rate. Our follow-up period was too short to evaluate the effects of our protocol on this tumor.

In the other 13 patients with intermediate prognosis, the total tumor-free rate of $61.5 \%$ after the initial treatment was significantly worse than that for germinoma (92.9\%). No recurrence during a median follow-up period of 2.6 years, however, is superior to the recurrence rate of approximately $30 \%$ in the 17 cases treated by radiation therapy alone in the University of Tokyo series[14] or to the median survival of 2 years in 21 previously reported cases (Table 4). 


\begin{tabular}{|c|c|c|c|c|}
\hline \multicolumn{5}{|c|}{$\begin{array}{c}\text { TABLE } 4 \\
\text { SUMMAY OF REPORTED CASES WITH INTERMED ATE PROGNOSIS TREATED } \\
\text { BV RADATION THERAPY }\end{array}$} \\
\hline Authors \& Year & $\begin{array}{l}\text { Patient } \\
\text { Age (yrs), } \\
\text { Sex }\end{array}$ & $\begin{array}{l}\text { Radation } \\
\text { Dose [Gy] }\end{array}$ & $\begin{array}{l}\text { His- } \\
\text { tology }\end{array}$ & $\begin{array}{l}\text { Last Report ed } \\
\text { Outcome }\end{array}$ \\
\hline $\begin{array}{l}\text { Fults \& Kelly, } 1983 \\
\text { Tajika, et al., } 1984 \\
\text { Allen, et al., } 1985 \\
\text { Nakamura, et al., } 1985 \\
\text { Sam ii, et al., } 1985 \\
\text { Shork y, et al., } 1985\end{array}$ & $\begin{array}{r}11, \mathrm{~F} \\
8, M \\
14, M \\
18, M \\
18, M \\
13, M \\
15, \mathrm{~F} \\
15, M \\
20, M \\
27, M \\
19, M \\
12, M \\
14, M \\
21, M \\
8, M \\
8, F \\
13, F \\
7, F \\
23, M \\
10, M \\
8, M\end{array}$ & $\begin{array}{r}45 \\
50 \\
40 \\
40 \\
58 \\
42 \\
35 \\
51 \\
50 \\
N D \\
35 \\
87 \\
60 \\
N D \\
48 \\
60 \\
55 \\
60 \\
60 \\
56 \\
35\end{array}$ & $\begin{array}{l}M X B \\
M T \\
M T \\
M X B \\
M T \\
M G T \\
M X B \\
M X B \\
M T \\
M T \\
M X B \\
M X B \\
M G T \\
M T \\
M T \\
M G T \\
M T \\
M T \\
M G T \\
M X B\end{array}$ & $\begin{array}{l}\text { alive, } 16 \text { mos } \\
\text { recurred, } 6 \text { mos } \\
\text { dead, } 12 \mathrm{mos} \\
\text { dead, } 2 \text { yrs } \\
\text { alive, } 3 \text { yrs } \\
\text { alive, } 3 \text { yrs } \\
\text { dead, } 5 \mathrm{mos} \\
\text { dead, } 2.6 \mathrm{yrs} \\
\text { dead, } 14 \mathrm{mos} \\
\text { alive, } 5.2 \mathrm{yrs} \\
\text { alive, } 3.5 \mathrm{yrs} \\
\text { alive, } 2.5 \mathrm{yrs} \\
\text { dead, } 1.5 \mathrm{yrs}\end{array}$ \\
\hline \multicolumn{5}{|c|}{$\begin{array}{l}\text { "MGT = mixed tumor with germinoma and teratoma } \\
\text { malignant teratoma; MXB = mixed tumor mainly consist } \\
\text { teratoma; } N D=\text { not described. }\end{array}$} \\
\hline
\end{tabular}

We could not control tumors in the patients with a poor prognosis. The tumors of three patients progressed before completion of initial treatment. Based on these unfortunate results, we have modified the protocol so that chemotherapy and radiation therapy are delivered simultaneously.

In conclusion, our multidisciplinary treatment contributes to a longer survival time with better quality life for patients with germinomas or those with an intermediate prognosis.

\section{References}

1. Allen JC, Bosl G, Walker R: Chemotherapy trials in recurrent primary intracranial germ cell tumors. J Neurooncol 3:147-152, 1985

2. Bosl GJ, Yagoda A, Whitmore WF Jr, et al: VP-16-213 and cisplatin in the treatment of patients with refractory germ cell tumours. Am J Clin Oncol 7:327-330, 1984

3. Chang CG, Kageyama N, Kobayashi T, et al: Pineal tumors: clinical diagnosis, with special emphasis on the significance of pineal calcification. Neurosurgery 8:656-668, 1981

4. Dattoli MF, Newall J: Radiation therapy for intracranial germinoma: the case for limited volume treatment. Int J Radiat Oncol Biol Phys 19:429-433, 1990

5. Einhorn LH, Donohue J: Cis-diamminedichloroplatinum, vinblastine, and bleomycin combination chemotherapy in disseminated testicular cancer. Ann Intern Med 87:293-298, 1977 
6. Fults D, Kelly DL Jr: A suprasellar atypical teratoma presenting as an intrasellar mass: a case report. Neurosurgery 13:40-43, 1983

7. Jenkin D, Berry M, Chan H, et al: Pineal region germinomas in childhood. Treatment considerations. Int J Radiat Oncol Biol Phys 18:541-545, 1990

8. Jennings MT, Gelman R, Hochberg F: Intracranial germ cell tumors: natural history and pathogenesis. J Neurosurg 63:155-167, 1985

9. Kida Y, Kobayashi T, Yoshida J, et al: Chemotherapy with cisplatin for AFP-secreting germ-cell tumors of the central nervous system. J Neurosurg 65:470-475, 1986

10. Matsukado Y, Abe H, Tanaka R, et al: [Cisplatin, vinblastine, and bleomycin (PVB) combination chemotherapy in the treatment of intracranial malignant germ cell tumors--a preliminary report of a phase II study. The Japanese Intracranial Germ Cell Tumor Study Group.] Gan No Rinsho 32:1387-1393, 1986 (Jpn)

11. Matsutani M, Asai A, Fujimaki T, et al: Successful treatment of recurrent malignant germ cell tumors: report of two cases. Neurosurgery 33:901-906, 1993

12. Matsutani M, Sano K, Takakura K: Long-term follow-up of patients with primary intracranial germinomas, in Packer R, Bleyer WL, Pochedly C (eds): Pediatric Neuro-oncology. Paris: Harwood, 1992, pp 254-260

13. Matsutani M, Sano K, Takakura K, et al: Combined treatment with chemotherapy and radiation therapy for intracranial germ cell tumors. Childs Nerv Syst 14:59-62, 1998

14. Matsutani M, Sano K, Takakura K, et al: Primary intracranial germ cell tumors: a clinical analysis of 153 histologically verified cases. J Neurosurg 86:446-455, 1997

15. Mineura K, Sasajima J, Sakamoto T, et al: [Treatment of intracranial germ cell tumors.] Gan No Rinsho 36:2399-2403, 1990 (Jpn)

16. Nakamura Y, Sato T, Nishimura G, et al: Malignant teratoma in the brain. An immunohistochemical study. Cancer 55:103-107, 1985

17. Neuwelt EA, Frenkel EP, Smith RG: Suprasellar germinomas (ectopic pinealomas): aspects of immunological characterization and successful chemotherapeutic responses in recurrent disease.

Neurosurgery 7:352-358, 1980

18. Ng HK, Poon WS, Chan YL: Basal ganglia teratomas: report of three cases. Aust NZ J Surg 62:436-440, 1992

19. Preissig SH, Smith MT, Huntington HW: Rhabdomyosarcoma arising in a pineal teratoma. Cancer 44:281-284, 1979

20. Rappaport R, Brauner R: Growth and endocrine disorders secondary to cranial irradiation. Pediatr Res 25:561-567, 1989

21. Sakata K, Yamada H, Sakai N, et al: Extraneural metastasis of pineal tumor. Surg Neurol 3:49-54, 1975 
22. Samii M, Ramina R, Koch G, et al : Malignant teratoma of the optic nerve: case report. Neurosurgery 16:696-700, 1985

23. Sawamura Y, Shirato H, Ikeda J, et al: Induction chemotherapy followed by reduced-volume radiation therapy for newly diagnosed central nervous system germinoma. J Neurosurg 88:66-72, 1998

24. Schulte FJ, Hermann HD, Muller D, et al: Pineal region tumours of childhood. Eur J Pediatr 146:233-245, 1987

25. Shibamoto Y, Abe Y, Yamashita J, et al: Treatment results of intracranial germinoma as a function of the irradiated volume. Int J Radiat Oncol Biol Phys 15:285-290, 1988

26. Shokry A, Janzer RC, von Hochstetter AR, et al: Primary intracranial germ-cell tumors. A clinicopathological study of 14 cases. J Neurosurg 62:826-830, 1985

27. Simson LR, Lampe I, Abell MR: Suprasellar germinomas. Cancer 22:533-544, 1968

28. Sugiyama K, Uozumi T, Arita K, et al: Clinical evaluation of 33 patients with histologically verified germinoma. Surg Neurol 42:200-210, 1994

29. Tajika Y, Kubo O, Himuro H, et al: [Clinico-pathological features of intracranial germ cell tumors with dissemination or metastasis in childhood.] No Shinkei Geka 12:161-168, 1984 (Jpn)

30. Uematsu Y, Tsuura Y, Miyamoto K, et al: The recurrence of primary intracranial germinomas. Special reference to germinoma with STGC (syncytiotrophoblastic giant cell). J Neurooncol 13:247-256, 1992

31. Yoshida J, Sugita K, Kobayashi T, et al: Prognosis of intracranial germ cell tumours: effectiveness of chemotherapy with cisplatin and etoposide (CDDP and VP-16). Acta Neurochir 120:111-117, 1993

Manuscript received May 18, 1998.

Accepted in final form June 24, 1998.

This work was supported by a Research Grant for Cancer (7-39 and 9-12) from the Japanese Ministry of Health and Welfare.

Address reprint requests to: Masao Matsutani, M.D., Department of Neurosurgery, Saitama Medical School, Morohongo 38, Moroyama, Irumagun Saitama, 350-04 Japan. email:

matutani@saitama-med.ac.jp. 